\title{
JÓVENES UNIVERSITARIOS: DESIGUALDADES SOCIOCULTURALES Y DIVERSIDAD DE REPRESENTACIONES EN TORNO A LA PROFESIÓN
}

\author{
Silvia Leticia Piñero Ramírez
}

Resumen: Se presenta un estudio de caso de las representaciones sociales que, sobre su profesión, tienen los jóvenes de nuevo ingreso a las carreras de Enfermería y de Médico Cirujano de la Universidad Veracruzana (U.V.). Mi hipótesis central es que el origen social de los estudiantes contribuye a configurar las anticipaciones y expectativas profesionales y, por lo tanto, su elección de carrera. El objetivo consiste en establecer si el conjunto de informaciones, creencias, opiniones y actitudes hacia la carrera que eligieron los jóvenes, se asocian con sus volúmenes y tipos de capital cultural. En este caso, la elección de carrera se explicaría desde una dimensión sociocultural, ligada a las estructuras de oportunidades. Se caracteriza a los jóvenes según su volumen de capital cultural y se establecen asociaciones estadísticas entre éste y una tipología construida de representaciones sociales. Los resultados permiten distinguir tipos de representaciones entre los universitarios, mismas que se confrontan con su capital cultural y la carrera elegida.

Palabras clave: Representaciones sociales, orígenes sociales, elección de carrera, estudiantes universitarios, capital cultural, identidad, profesiones.

Enviado a dictamen: 19 de nero de 2012

Aprobación: 11 de mayo de 2012

Revisiones: 1

Mtra. Silvia Leticia Piñero Ramírez, profesora de la Facultad de Pedagogía, Universidad Veracruzana, Maestra en investigación educativa, estudiante del Doctorado en Investigación educativa del Instituto de Investigaciones en Educación, Universidad Veracruzana. Temas de investigación: desigualdades socioeconómicas y culturales y sus efectos en la oportunidad educativa; prácticas culturales de estudiantes; representaciones sociales como instrumento para explicar y comprender las elecciones profesionales. Correo electrónico: spinero@uv.mx.
Abstract: We present a case study of social representations on their profession, have incoming young people to careers in nursing and Surgeon of the Universidad Veracruzana (UV). My hypothesis is that the social origin of students contributes to shaping the professional expectations and anticipations and therefore your choice of career. The aim is to establish whether the set of information, beliefs, opinions and attitudes of young people who chose to race, are associated with the volumes and types of cultural capital. In this case, the choice of career be explained from a socio-cultural dimension, linked to opportunity structures. Youth is characterized by volume of cultural capital and establish statistical associations between it and built a typology of social representations. The results allow to distinguish types of representations between the university, which are being confronted with their cultural capital and their chosen career.

Keywords: Social representations, social origin, career choice, college students, cultural capital, identity, professions.

\section{Introducción}

E l enfoque vocacional, que ha dominado los trabajos científicos y las prácticas de orientación en las escuelas de nivel medio superior o bachilleratos, privilegia una supuesta disposición casi genética de los intereses y motivaciones que conducen a la elección de carrera. Este enfoque ignora, 
en cambio, las fuerzas sociales que condicionan las anticipaciones y expectativas de los jóvenes hacia las carreras profesionales.

Este trabajo analiza la elección profesional desde una perspectiva sociocultural que incorpora el análisis de los rasgos estructurales de los jóvenes y de sus influencias sobre las elecciones y las prácticas profesionales, entendidas como formas de acción social. Nos aproximamos a la forma en que se configuran las elecciones y las prácticas ligadas a la profesión, en interacción con elementos que definen la estructura social (Berger y Luckmann, 1998).

Una de las principales motivaciones para realizar este estudio provino de observar un fenómeno reiterado en algunas carreras a nivel nacional y en la propia U.V., en particular, Enfermería y Médico Cirujano. Nos referimos a una fuerte competencia entre los jóvenes por conseguir un lugar en ambas que es más acentuada en la última.

En México es elevada la tendencia de los jóvenes a solicitar ingreso en las carreras del campo de la Medicina, hecho que las coloca como objeto de interés analítico. En el año 2010, nueve de cada cien jóvenes solicitaron ingreso a esta carrera en universidades públicas del país, si bien, en 15 estados esta proporción fue mayor. En Chihuahua treinta de cada cien jóvenes solicitaron ingreso a universidades públicas en esta carrera. Por su parte, tres de cada cien jóvenes solicitaron ingreso a Enfermería en el país, siendo el estado de Guerrero donde se registra la proporción más alta, pues nueve de cada cien solicitaron ingreso a esta carrera en universidades públicas (Universidad Veracruzana-Secretaría de Educación Pública, 2012).

Ese mismo año la Universidad Veracruzana acapara 78. $6 \%$ de la demanda a la carrera de Enfermería en universidades públicas del estado de Veracruz. A la vez, concentra la demanda total de aspirantes a la carrera de Medicina en el mismo tipo de institución. En el año 2007, año en que se realizó este estudio, de un total de 40,444 jóvenes, $4.7 \%$ de los aspirantes a la U.V. solicitó ingreso a la carrera de Enfermería, mientras que $11.5 \%$ lo hizo a la de Médico Cirujano (Universidad Veracruzana, 2007).

Los orígenes socioeconómicos y culturales de los jóvenes que ingresan a Enfermería o a Médico Cirujano son notablemente desiguales entre ambas carreras. El trabajo reportado por Colorado (2008) corrobora la heterogeneidad de los rasgos que definen la diversidady desigualdad socioeconómica y cultural entre los jóvenes que ingresan a tales carreras.

El objetivo central de este trabajo es explicar algunas tendencias en las elecciones de carrera a partir de las representaciones de los jóvenes sobre la profesión, incorporando como elemento mediador de la elección los condicionamientos estructurales de las acciones. Jon Elster se refirió a éstos así: "aun cuando el comportamiento es el resultado de deseos y oportunidades, su varianza a lo largo del tiempo puede explicarse en gran medida sobre la base de las segundas" (Elster, 2007: 189). La inclinación de muchos jóvenes a elegir la Medicina como su carrera y futura profesión se liga al marco percibido de oportunidades que los jóvenes se han construido por sí mismos a lo largo de su trayectoria biográfica, lo que que permite explicar las tendencias de los jóvenes a concentrar su elección en un reducido número de carreras, dependiendo de su origen social.

Por lo tanto, dos son los ejes en que se asienta este estudio: el capital cultural de los jóvenes, que sintetiza conceptualmente y define operacionalmente el origen socioeconómico y cultural de los estudiantes de nuevo ingreso de las carreras de Enfermería y de Médico Cirujano. El segundo eje se centra en las representaciones en torno a la profesión a la que ingresaron los estudiantes de nuevo ingreso. A partir de la teoría de las representaciones sociales intentamos explicar un rostro no explorado de la elección profesional: ¿Qué anticipaciones y expectativas elementos de representación - dominan entre jóvenes de escasos recursos económicos y culturales? ¿Cómo difieren aquéllas de las 
anticipaciones y expectativas de jóvenes con abundancia de recursos económicos y materiales?

El supuesto inicial que asumimos es que las representaciones sociales de los jóvenes en torno a las profesiones son compartidas dentro de un marco de referencia común: su origen social (Doise, et al., 2005). El segundo supuesto sostiene que las representaciones sociales mantienen una función guiadora de las prácticas de los jóvenes y de las personas en general (Moscovici, 1979; Abric, 2001; Doise, et al., 2005). Por ello, la elección de carrera profesional encuentra un fuerte sustento en las representaciones profesionales (Blin, 1997).

\section{Formulación del problema}

La perspectiva psicopedagógica dominante recupera una definición de la elección profesional como un acto meditado con base en el conocimiento de las opciones profesionales existentes, mientras un enfoque vocacional apunta al reconocimiento de las capacidades propias para las diversas opciones profesionales. Más allá de estas visiones consideramos una tercera opción: la posibilidad de que en la elección de carrera intervengan tanto aspectos conscientes para los jóvenes - la existencia y el conocimiento de diversas opciones profesionales y el reconocimiento de las capacidades propias para desempeñarla- como elementos inconscientes que entran en el terreno de lo común y dado al agente, el habitus ${ }^{1}$ (Bourdieu, 2002).

Nuestro interés es conocer la manera en que las representaciones sociales acerca de las profesiones constituyen formas simbólicas que los jóvenes tienen incorporadas en virtud de su habitus y guían las disposiciones de los jóvenes hacia determinada(s) carrera(s).

El conjunto "de informaciones, de creencias, de opiniones y de actitudes a propósito de un objeto dado" (Doise, et al., 2005) —en este caso la profesiónnos permite aproximarnos a los aspectos que entran en juego cuando los jóvenes eligen una carrera. En particular nos interesa destacar el papel de la condición socioeconómica y cultural de los jóvenes, que se encuentra enraizada en sus antecedentes familiares y escolares.

\section{El anclaje teórico \\ Los jóvenes desde un enfoque sociocultural}

Desde una perspectiva sociocultural incorporamos el papel que ejerce la estructura de oportunidades de los jóvenes en la elección profesional. Los intercambios que los jóvenes mantienen con las instituciones y grupos sociales -incluyendo, la familia, la escuela y el vecindario (Furstenberg, 2006) - contribuyen a configurar esta estructura.

Las condiciones de vida de los jóvenes delimitan el acceso que tienen a los recursos materiales o simbólicos, provistos en el seno de los contextos más extensos y de los más inmediatos, imponiendo un límite a sus posibilidades de interacción social. Para los jóvenes estas restricciones propician un diferencial acceso a medios de socialización y de adquisición o intercambio de recursos culturales a través de la familia, los amigos, los vecinos, los profesores y los pares, promoviendo o disminuyendo sus posibilidades de logro académico (Furstenberg, 2006). La autopercepción que los jóvenes tienen de su propia capacidad de logro incide en sus anticipaciones, elecciones y expectativas de futuro.

De acuerdo con Gilberto Giménez "el paradigma de las representaciones sociales [...] permite detectar esquemas subjetivos de percepción, de valoración y de acción que son la definición misma del habitus bourdieusiano y de lo que nosotros hemos llamado cultura interiorizada [las cursivas son nuestras]" (Giménez, 2005a: 16). Por ello la elección de carrera sintetiza, más allá de una toma de posición formativa, profesional y ocupacional, la expresión de elementos culturales que contribuyen a definir la identidad de los jóvenes. 
En otro manuscrito Giménez sostiene: "la identidad no es más que la cultura interiorizada por los sujetos, considerada bajo el ángulo de su función diferenciadora y contrastiva en relación con otros sujetos" (Giménez, 2005b:5) Lasinteracciones de los jóvenes en contextos de oportunidad diferenciados contribuyen a la formación de un sentido de pertenencia y a la conformación de una identidad grupal. La elección de carrera profesional responde así a una identificación con grupos de pares que se anticipan en el futuro a pesar de que no existen certezas para los jóvenes en torno a su futuro ocupacional.

\section{Representaciones, habitus y posición social}

En torno a la elección profesional suele producirse un abanico de anticipaciones y expectativas a mediano y largo plazo centradas en la utilidad económica que los jóvenes consideran que obtendrán a través de su profesión, mientras que otro tipo de anticipaciones y expectativas tienen que ver con su vida personal.

La posición de los jóvenes en el espacio social ejerce una influencia en la perspectiva desde la cual construye su realidad. Su ubicación en el interior de un campo y en un espacio social determinado ejerce una gran influencia en la forma en que se apropia de cierta cantidad y tipos de información. En este proceso de intercambios sociales las representaciones se insertan como formas de pensamiento producidas en contextos específicos.

A cada espacio social corresponden formas específicas de distribución de los recursos económicos, sociales, culturales y simbólicos. Al asignarle significados simbólicos el agente participa en la construcción de esa estructura social: el habitus cumple un papel legitimador de las prácticas del agente. Bourdieu señala:

Aprehende las diferencias de condición, que retiene bajo la forma de diferencias entre unas prácticas enclasadas y enclasantes (como productos del habitus), según unos principios de diferenciación que, al ser producto de estas diferencias, son objetivamente atribuidos a éstas y tienden por consiguiente a percibirlas como naturales". (Bourdieu, 2002: 171)

Los modos en que el agente se apropia de la realidad configuran el habitus. Las representaciones juegan un papel determinante en la configuración del habitus, dado que constituyen los lentes figurativos a través de los cuales el agente re-construye los elementos que lo definen.

Las representaciones sociales se encuentran determinadas por la pertenencia social del agente, como señalan Willem Doise, Alain Clémence y Fabio LorenziCioldi: "Si [...] pensamos que las representaciones son ante todo principios organizadores que regulan las relaciones simbólicas, podemos aceptar que esta organización genera diferencias y variaciones sistemáticas entre individuos" (Doise, et al., 2005: 95). Así, las condiciones de vida y relaciones sociales de los jóvenes, definen su diversidad de representaciones sociales y su carácter compartido (Moscovici, 1979).

Al hablar de las funciones que desempeñan las representaciones sociales, Abric escribe:

La representación produce igualmente un sistema de anticipaciones y expectativas. Es así, pues, una acción sobre la realidad: selección y filtro de informaciones, interpretaciones con objeto de volver esa realidad conforme a la representación. La representación por ejemplo no sigue; depende de la evolución en una interacción: la precede y determina [las cursivas son del original]. (2001: 16)

Al analizar las representaciones de la profesión podemos aproximarnos a tales anticipaciones y expectativas, que son construidas socialmente y desembocan en la elección de carrera.

El mismo autor apuntó a propósito de la elección profesional: "se admite en general que las 
representaciones sociales de los actores desempeñan un papel importante en [...] [la] orientación [del problema de la elección profesional]" (Abric, 2001: 203). Por lo tanto, la perspectiva de las representaciones sociales, proporciona un puente que permite analizar la elección profesional, considerando que la subjetividad de la persona es construida socialmente (Berger $\&$ Luckmann, 1998).

El capital cultural: expresión de la posición social de los jóvenes

Pierre Bourdieu conceptualizó el capital cultural como la posesión de atributos culturales que expresan las posiciones de los agentes en el espacio social. El concepto es útil para adentrarse en el reconocimiento de las posiciones sociales de los jóvenes que eligen carreras profesionales. Al explicar que el capital cultural es una expresión materializada del espacio social en que se ubica el sujeto, Bourdieu enfatiza el papel que desempeña el contexto sociocultural en la elección de una determinada carrera.

El capital cultural y el capital económico de los agentes puede ser medido en su estructura y en su volumen; la medida resultante permite definir criterios de diferenciación social entre los jóvenes (Bonnewitz, 2003). El volumen y la estructura del capital se configuran a partir de la ubicación que ocupa el agente dentro del espacio social, si bien, su adquisición y aumento, contribuye a modificar dicha posición.

El capital es el conjunto de medios materiales y simbólicos cuyo punto de partida es la herencia familiar, que configuran la manera en que los jóvenes pueden tener acceso a una diversidad de otros bienes materiales o simbólicos. Estos medios pueden expresarse en la incorporación de atributos subjetivos en forma de cualidades, gustos y preferencias (Cfr. con Bourdieu, 2002). Este concepto se refiere así, no sólo a la posesión de bienes culturales ligados a la escolaridad, sino que incluye el dominio y beneficio de los recursos materiales de tipo económico de origen familiar.
Bourdieu y Passeron mostraron que el origen social de los estudiantes ejerce una influencia mediadora en todos los ámbitos de la vida de las personas al señalar:

El origen social es, de todos los determinantes, el único que extiende su influencia a todos los dominios y todos los niveles de la experiencia de los estudiantes, y en primer lugar a sus condiciones de existencia. El hábitat y el tipo de vida cotidiano que le está asociado, el aumento de recursos y su reparto entre las diferentes partidas presupuestarias, la intensidad y la modalidad del sentimiento de dependencia, variable según el origen de los recursos así como según la naturaleza de la experiencia y los valores asociados a su adquisición, dependen directa y fuertemente del origen social al mismo tiempo que refuerza su eficacia. (Bourdieu \& Passeron, 2003: 26-27).

El capital cultural, en tanto define la posesión de bienes culturales materiales o simbólicos, puede ser definitorio en el acceso que tienen los jóvenes a otras oportunidades, escolares, institucionales, sociales; del mismo modo puede ser determinante del ingreso de los jóvenes a las instituciones de educación superior.

\section{Aspectos metodológicos Población}

El estudio se limitó a la población de estudiantes de primer semestre de las carreras de Enfermería y de Médico Cirujano de todos los campus de la U.V., que suman 944 jóvenes.

Ejes de análisis, criterios de operacionalización y datos

a) El capital cultural de los jóvenes

Elprimer eje se operacionalizó a través de los indicadores propuestos por Casillas, et al., 2007, para el estudio y diferenciación de los orígenes sociales de los aspirantes (ver cuadro 1). 
Con el fin de establecer grupos de estudiantes diferenciables entre sí conforme a su origen social, se analizaron las variables con el método de conglomerados o cluster analysis. Este método permite rastrear patrones en los datos, agrupando individuos en conjuntos con sentido conceptual y empírico. ${ }^{2}$ Según Steinbach "el conglomerado se puede mirar como forma de clasificación que crea un etiquetado de objetos con las etiquetas de la clase (clúster)" (Steinbach et al., 2006: 490).Por ello el método requiere etiquetar a los grupos de acuerdo con los rasgos encontrados. Los análisis fueron realizados con el paquete Statistica.

Los datos para la construcción de la tipología de capital cultural fueron tomados de la encuesta que la U.V. aplica a los aspirantes cuando solicitan el Examen Nacional de Ingreso a la Educación Superior (EXANIII). En ella se recopila información socioeconómica, cultural, demográfica y antecedentes escolares de los aspirantes a la U.V.

\section{b) Las representaciones sociales en torno a la profesión de adscripción}

Las representaciones de la profesión se definieron a partir de las principales dimensiones analíticas consideradas en la literatura especializada en el estudio de las profesiones. Se construyó y aplicó un cuestionario tipo escala Likert, que indujo en los jóvenes el reconocimiento de los principios que organizan sus representaciones sociales. Estas expresan un conjunto de "información, valores, opiniones, actitudes, normas y creencias" (Doise, et al., 2005: 12).

Las dimensiones analíticas de la profesión recuperadas por el cuestionario son: formación, finalidad, beneficios, status y prestigio, desempeño laboral, criterios de satisfacción y movilidad (ver cuadro 2).

Se realizó otro análisis de conglomerados, ahora para identificar rasgos de representaciones entre los aspirantes de ambas carreras, a modo de establecer grupos de jóvenes diferenciables que permitieran elaborar tipologías.

Una vez establecidas las tipologías de capital cultural y de representaciones de la profesión, se establecieron asociaciones entre estas dimensiones.

\section{Resultados}

\section{a) Capital cultural de los estudiantes de Enfermería y de Médico Cirujano}

Un rasgo común entre los jóvenes de ambas carreras son sus altos volúmenes de capital escolar. Esto significa que sus resultados académicos, medidos a través de la trayectoria en el bachillerato, son similares (nivel medio alto), como lo muestra la gráfica 1.

En lo que respecta al capital familiar, la mayoría de los estudiantes de nuevo ingreso de Enfermería es muy pobre o pobre, mientras que la mayoría de los jóvenes de la carrera de Médico Cirujano provienen de familias con un nivel elevado de recursos económicos. Por lo tanto, esta variable estableció diferencias plenas entre los estudiantes de ambas carreras, visibles a través de la conformación de tres clúster o grupos observables en las líneas expresadas en la gráfica 1 .

Los grupos se etiquetaron del siguiente modo, adaptando la propuesta de Casillas et al., 2007.

Clúster 1 (Pobres exitosos): agrupa a los jóvenes que tienen un capital escolar medio alto y un capital familiar bajo. Su trayectoria escolar es elevada, si bien su condición socioeconómica es baja, y sus padres tienen una escolaridad básica o de nivel medio superior.

Clúster 2(Herederos): agrupa a los jóvenes que tienen un capital escolar medio alto y un capital familiar medio alto. Con una trayectoria escolar elevada, se diferencian del grupo anterior por su solvencia económica y porque la escolaridad de sus padres es de nivel superior.

Clúster 3 (Héroes): agrupa a los jóvenes que tienen un capital escolar medio o alto, y su capital familiar es bajo. 
Las diferencias entre estos grupos expresan no solo desigualdades socioeconómicas, culturales y escolares sino ventajas o desventajas académicas que se reflejan en los modos en que los jóvenes transitan sus estudios superiores, distintos para los jóvenes de cada grupo.

El 70\% de los jóvenes que ingresaron a Enfermería en el año 2007 son de capital cultural muy pobre (Héroes) o pobre (Exitosos), mientras que 65\% de quienes ingresaron a Médico Cirujano tienen un capital cultural alto (Herederos) (ver cuadro 3).

\section{b) Representaciones de las profesiones entre los estudiantes de} Enfermeríay de Médico Cirujano

El análisis de clúster permitió diferenciar tres grupos de jóvenes según la medida en que comparten determinados elementos de las representaciones profesionales (gráfica 2). Estos elementos diferenciadores nos permitieron elaborar tipologías de representaciones. Estas tipologías se etiquetaron a modo de identificar los rasgos más sobresalientes que definen la diferencias entre cada grupo, de acuerdo con las dimensiones del cuadro 2 ya presentado. La gráfica 2 muestra tres grupos de jóvenes que se etiquetaron del siguiente modo: (ver gráfica 2).

Clúster l (filántropos privados). Los principios que organizan sus representaciones son:

- Una elevada finalidad social de la profesión que define los rasgos filantrópicos de estos jóvenes.

- Los criterios de satisfacción se centran en el apoyo que se brinda a la gente, en el mejoramiento de la sociedad y en el desarrollo personal y de las competencias técnicas propias. Se desligan los criterios económicos en la definición de la profesión que también define rasgos de filantropía.

- La formación se orienta a la adquisición de habilidades, a la autonomía y la flexibilidad.
- La utilidad privada se define en función del desarrollo personal y se opone a los beneficios económicos.

- Seniega la posibilidad de una movilidad económica ascendente, aunque se reconoce cierto aumento del status social.

- El desempeño laboral se define mediante el trabajo por cuenta propia. Prevalece incertidumbre sobre la posibilidad de un trabajo bien pagado y de que los profesionistas alcancen la autosuficiencia económica a través del mismo.

- Ausencia de reconocimiento del status y prestigio de la profesión.

Clúster 2 (generosos escépticos). En general las representaciones de este grupo no se apegan de manera firme a las dimensiones propuestas, como se muestra en los bajos promedios que adquiere cada dimensión representada en la gráfica 2. No obstante, los rasgos que definen sus representaciones, se organizan bajo los siguientes principios:

- La finalidad social de la profesión.

- Los criterios de satisfacción provienen del apoyo brindado a la gente en el desarrollo personal y se apartan de criterios económicos. De aquí viene en parte la etiqueta de generosos.

- La formación profesional secentra en las habilidades y se aleja de la imagen de un desempeño laboral autónomo y con flexibilidad laboral.

- La utilidad privada se orienta al desarrollo personal y se aparta de los beneficios económicos derivados del desempeño profesional, definiendo también rasgos de generosidad.

- Moderado reconocimiento del status y prestigio de la profesión.

- Limitado reconocimiento de los rasgos que definen el desempeño laboral.

- Se niega en absoluto la posibilidad de movilidad social y económica ascendente, hecho que 
contribuye a destacar el escepticismo de la profesión como medio para la mejora económica y social, individual y familiar.

Clúster 3 (altruistas ostensibles). Las representaciones de este grupo se definen así:

- Una sólida finalidad social de la profesión.

- Un alto status y prestigio de la profesión, a través del cual, se vislumbra un aire de prominencia profesional y social.

- Los criterios de satisfacción altamente definidos por el carácter humanitario de la profesión y su contribución al desarrollo personal y de las competencias técnicas del profesionista. De ahí la etiqueta altruista que define a este grupo.

- La utilidad privada, orientada al desarrollo personal y la independencia personal, apunta a la oportunidad para encontrar un buen puesto de trabajo. Se reconocen beneficios derivados del ingreso económico.

- La formación se orienta a la adquisición de habilidades, a la autonomía y la flexibilidad laboral.

- El reconocimiento de la movilidad social y económica ascendente, vinculada a la adquisición de prominencia a través de la profesión.

- Moderado apego a los rasgos que definen el desempeño laboral, según la imagen de un profesionistaautónomolaboralyeconómicamente, sin que ello implique un ingreso elevado.

c) Asociaciónentre el capital cultural y las representaciones de las profesiones en los estudiantes de Enfermería y Medicina

Al analizar la asociación entre capital cultural y las tipologías de representaciones, por carreras, encontramos los datos presentados en el cuadro 4. De acuerdo con éstos, sostenemos que un típico joven de nuevo ingreso a la carrera de Enfermería, tiene una representación filantrópica de la profesión basada en la ayuda que se brinda al otro y al mejoramiento de la sociedad. Niegan los beneficios económicos, cuestionan la posibilidad de una movilidad económica, pero reconocen la posibilidad de un aumento del status social de la familia y niegan el reconocimiento social de la profesión. Son mayoritariamente filántropos privados, pues recordemos que los héroes predominan entre quienes ingresaron a la carrera de Enfermería en el año 2007 (ver cuadro 4).

El cuadro 5 presenta los datos del cruce entre capital cultural y representaciones en los estudiantes de nuevo ingreso de Medicina. Un típico joven héroe de esta carrera tiene una imagen altruista, ligada con ciertos beneficios económicos y la posibilidad de un ascenso económico y social, y reconoce en ella su status y prestigio (altruistas ostensibles). O bien, comparten una imagen generosa que niega los beneficios económicos y la movilidad social y económica ascendente. No obstante, reconoce el status y el prestigio de la profesión (generosos escépticos). Recordemos nuevamente que los herederos son el grupo de estudiantes de nuevo ingreso que predomina en el año 2007 (ver caudro 5).

\section{Conclusiones}

Hemos mostrado que los orígenes sociales de los jóvenes constituyen un medio de aproximación a la elección de carrera profesional Para entenderlo recurrimos al análisis de los principios que organizan sus representaciones sobre las profesiones. Estasúltimas expresan elementos de identidad de los jóvenes situados en espacios socioeconómicos y culturales diferenciados, como también expresan tomas de posición al interior del campo de las profesiones.

Como fue mostrado, en los jóvenes de la carrera de Enfermería, prevalece una representación filantrópica, desinteresada en un sentido económico de la profesión. Mientras que en la carrera de Médico Cirujano prevalece una representación generosa que, de hecho, reconoce 
el carácter humanitario de la labor profesional pero a la vez, sobredimensiona el status y el prestigio de la profesión, y aspira a una movilidad social y económica ascendente, en jóvenes que, de por sí, gozan de una condición privilegiada.

Las tipologías de las representaciones profesionales, expresan los códigos dominantes del grupo o los grupos sociales a que pertenecen (Bernstein, 1989). El hecho de que entre los herederos, los pobres exitosos y los héroes prevalezcan diferentes representaciones expresa la existencia de creencias, informaciones y valores más o menos compartidos, aunque no sean homogéneos.

La pertenencia social de un agente es capaz de revelar la tendencia a compartir determinado tipo de representación profesional. Así, este tipo de representación en particular, adquiere un carácter dominante en el grupo. En este sentido consideramos que la estructura social contribuye a generar y reproducir relaciones sociales basadas en el discurso y la acción. Bernstein señaló: "la estructura social genera distintas formas lingüísticas o códigos, y estos códigos transmiten esencialmente la cultura y, de este modo, constriñen el comportamiento" (Bernstein, 1989: 130). La pertenencia a un grupo social, definida en este trabajo en función del capital cultural, expresa la tendencia a compartir cierto tipo de representación. Del mismo modo podría llevar a considerar que determinadas preferencias profesionales tienen relación con dicha pertenencia.

Lo presentado hasta ahora nos conduce a nuevos supuestos: ies acaso el origen social una de las condiciones que lleva a los jóvenes a reproducir las representaciones de la profesión, y por lo tanto, la carrera que eligen? Al mismo tiempo nos conduce a considerar si el contexto social en que viven, facilita las condiciones de producción y de reproducción de sus representaciones profesionales: ila supremacía de ciertas formas de pensamiento social contribuye a reproducir las relaciones de dominación en el ámbito profesional?
El análisis de los condicionamientos socioculturales de los jóvenes universitarios, alcanza el nivel de las subjetividades compartidas por los jóvenes. En este sentido es de utilidad reconocer los factores determinantes de la construcción de su proyecto personal y profesional.

\section{Notas}

${ }^{1}$ Definimos el habitus como el conjunto de disposiciones, usos y expresiones materiales y subjetivas de las condiciones de vida, formas de pensamiento y de los recursos materiales y simbólicos a los que el agente tiene acceso cotidianamente, en virtud de su pertenencia a determinado espacio social (Cfr. Bourdieu, 2002).

${ }^{2}$ Steinbach, Tan \& Kumar señalan sobre este método estadístico: El análisis de clúster agrupa datos de objetos basados sólo en la información encontrada en los datos que describen los objetos y sus relaciones. La meta es que los objetos dentro de un grupo sean similares (o relacionados) con los otros y diferentes de (o desligados de) los objetos de otros grupos. La más grande similitud (u homogeneidad) dentro de un grupo y la más grande diferencia entre los grupos es la mejor o mayor distinción entre los clúster (2006: 490).

${ }^{3}$ La propuesta original de Casillas, Chain y Jácome (2007), incluye la ocupación de los padres como un indicador de capital familiar. En este estudio no se consideró ese indicador por la ausencia de los datos.

\section{Bibliografía}

Abric, Jean C. (2001), Prácticas sociales y representaciones, (Tradicido por J. Dacosta y F. Flores), México: Coyoacán.

Berger, P. \& Luckmann, T. (1998), La construcción social de la realidad, Buenos Aires: Amozurrutu.

Bernstein, B. (1989), Clases, códigos y control I. Estudios para una sociología del lenguaje, Madrid: Akal Universitaria. 
Blin, J. F. (1997), Représentations, pratiques et identités professionnelles, París: Editions L'Harmattan.

Bonnewitz, P. (2003), La sociología de Pierre Bourdieu, Buenos Aires: Nueva Visión.

Bourdieu, P. (2002), La distinción. Criterios y bases sociales del gusto, España: Taurus.

Bourdieu, P. \& Passeron, J. Claude (2003), Los herederos. Los estudiantes y la cultura, Buenos Aires: Siglo XXI.

Casillas, M., Chain, R. \& Jácome, N. (2007), Origen social de losestudiantes y trayectorias estudiantiles en la Universidad Veracruzana. Revistadelaeducación superior, [enlínea] abril-junio, 142, pp. 7-29. Disponible en http: http:// redalyc.uaemex.mx/redalyc/pdf/604/60414201.pdf, [consultado: noviembre 26 de 2008].

Chain, R. et al. (2001), Demanda, estudiantes, y elección, Xalapa, Veracruz, México: Universidad Veracruzana.

COINPI (Consulta Individualizada de Perfil de Ingreso), (2007), [Disco Compacto]. Xalapa, Veracruz, México: Universidad Veracruzana.

Colorado, A. (2008), Estrategias de inversión y conversión del capital cultural y otros tipos de capital en la definición de las trayectorias escolares universitarias. El caso de los estudiantes de Medicina e Ingeniería Civil de la Universidad Veracruzana. Tesis de Maestría en Sociología. México: Universidad Autónoma Metropolitana.
Doise, W., Clèmence, A. \& Lorenzi-Cioldi, F. (2005), Representaciones sociales y análisis de datos, México: Instituto Mora.

Elster, J. (2007), La explicación del comportamiento social. Más tuercas y tornillos para las ciencias sociales, [Traducción de Horacio Pons], Barcelona, España: Gedisa.

Furnstenberg Jr, Frank F. (2006), “Diverging development: the not-so-invisible hand of social class in the United States", in Research Network on Transitions to Adulthood, Biennal meetings of the Society for Research on Adolescence, San Francisco, C.A. pp. 23-26.

Giménez, G. (2005a), capítulo I: La concepción simbólica de la cultura. [Manuscrito no publicado], en http:// www.paginasprodigy.com/peimber/cultura.pdf, [consultado: febrero 6 de 2008].

Giménez, G. (2005b), La cultura como identidad y la identidad como cultura, México: Consejo Nacional de la Cultura y las Artes., [consultado: mayo 72012 ].

Moscovici, S. (1979), El psicoanálisis, su imagen y su público, [Traducción de N. M. Finetti], Argentina: Huemul.

Universidad Veracruzana-Secretaría de Educación Pública (2012), Estadística del cuestionario 911 del año 2011, [medio electrónico], Xalapa, Veracruz, México.

Steinbach, M., Tan P.- N. \& Kumar, V. (2006), Introduction to Data Mining, USA: Pearson.

\section{Cuadros y gráficas}

Cuadro 1. Indicadores de capital cultural de los jóvenes de nuevo ingreso a Enfermería y Médico Cirujano, U.V., 2007

\begin{tabular}{|c||c||l||}
\hline \hline Dimensión & Variables & \multicolumn{1}{c|}{ Indicadores } \\
\hline \hline \multirow{3}{*}{ Capital cultural } & Capital familiar & $\begin{array}{l}\text { Escolaridad de los padres } \\
\text { Nivel socioeconómico familiar }\end{array}$ \\
\cline { 2 - 3 } & Capital escolar & $\begin{array}{l}\text { Promedio de calificaciones en el Bachillerato } \\
\text { Calificación obtenida en el examen de ingreso } \\
\text { Reprobados o no en el Bachillerato } \\
\text { Año de egreso del bachillerato }\end{array}$ \\
\hline
\end{tabular}

Fuente: Casillas, M., Chain, R. \& Jácome, N., 2007. 
Cuadro 2. Representaciones de la profesión de adscripción de los jóvenes de nuevo ingreso a Enfermería y Médico Cirujano, U.V., 2007

\begin{tabular}{|c|c|c|}
\hline Dimensión & Variables & Indicadores \\
\hline \multirow{7}{*}{$\begin{array}{l}\text { Representaciones } \\
\text { sociales de la } \\
\text { profesión }\end{array}$} & A. Formación & $\begin{array}{l}\text { a.1 Teórica/práctica } \\
\text { a.2 Especificidad } \\
\text { a.5 Áreas: } \\
\text { a.5.1 Personalidad } \\
\text { a.5.2 Conocimiento } \\
\text { a.5.3 Habilidades } \\
\text { a.5.4 Competencias }\end{array}$ \\
\hline & $\begin{array}{l}\text { B. Finalidad } \\
\text { social }\end{array}$ & $\begin{array}{l}\text { b.1 Sociedad } \\
\text { b.2 Trato profesional } \\
\text { b.3 Personas allegadas }\end{array}$ \\
\hline & $\begin{array}{l}\text { C. Utilidad } \\
\text { privada }\end{array}$ & $\begin{array}{l}\text { c.1 Personales } \\
\text { c. } 2 \text { Económicos } \\
\text { c.3 Laborales }\end{array}$ \\
\hline & $\begin{array}{l}\text { D. Status y } \\
\text { prestigio }\end{array}$ & $\begin{array}{l}\text { d.1 Status profesional } \\
\text { d.2 Reconocimiento social }\end{array}$ \\
\hline & $\begin{array}{l}\text { E. Desempeño } \\
\text { laboral }\end{array}$ & $\begin{array}{l}\text { e.1 Sector } \\
\text { e.2 Ingreso económico } \\
\text { e.3 Tiempo libre } \\
\text { e.4 Tipo de desempeño: } \\
\text { e.4.1 Habilidades } \\
\text { e.4.2 Conocimientos } \\
\text { e.4.3 Actitudes }\end{array}$ \\
\hline & $\begin{array}{l}\text { F. Criterios de } \\
\text { satisfacción }\end{array}$ & $\begin{array}{l}\text { f.1 Humanitarios } \\
\text { f.2 Personales } \\
\text { f.3 Técnicos } \\
\text { f.4 Económicos }\end{array}$ \\
\hline & G. Movilidad & $\begin{array}{l}\text { g.1 Movilidad social } \\
\text { g.2 Movilidad económica }\end{array}$ \\
\hline
\end{tabular}

Fuente: elaboración propia. 
Cuadro 3. Capital cultural de los estudiantes de primer ingreso a Enfermería y Médico Cirujano, U.V., 2007

\begin{tabular}{|c|c|c|c|}
\hline Capital cultural & & Enfermería & Médico Cirujano \\
\hline \multirow{2}{*}{ Héroes } & Absolutos & 246 & 76 \\
\hline & Relativos & $46.24 \%$ & $19.38 \%$ \\
\hline \multirow{2}{*}{ Pobres exitosos } & Absolutos & 130 & 61 \\
\hline & Relativos & $24.43 \%$ & $15.56 \%$ \\
\hline \multirow{2}{*}{ Herederos } & Absolutos & 156 & 255 \\
\hline & Relativos & $29.32 \%$ & $65.05 \%$ \\
\hline \multirow{2}{*}{ Total } & Absolutos & 532 & 392 \\
\hline & Relativos & $100 \%$ & $100 \%$ \\
\hline
\end{tabular}

Fuente: elaboración propia.

Cuadro 4. Tipología de representaciones de la profesión de los jóvenes de nuevo ingreso de la carrera de Enfermería. U.V., 2007

\begin{tabular}{|c|c|c|c|c|c|}
\hline $\begin{array}{c}\text { Representaciones } \\
\text { Capital cultural }\end{array}$ & & $\begin{array}{l}\text { 1. Filántropos } \\
\text { privados }\end{array}$ & $\begin{array}{l}\text { 2. Generosos } \\
\text { escépticos }\end{array}$ & $\begin{array}{l}\text { 3. Altruistas } \\
\text { ostensibles }\end{array}$ & Total \\
\hline \multirow{2}{*}{ Héroes } & Absolutos & 90 & 40 & 35 & 165 \\
\hline & Relativos & $54.55 \%$ & $24.24 \%$ & $21.21 \%$ & $100 \%$ \\
\hline \multirow{2}{*}{ Pobres exitosos } & Absolutos & 41 & 21 & 32 & 94 \\
\hline & Relativos & $43.62 \%$ & $22.34 \%$ & $34.04 \%$ & $100 \%$ \\
\hline \multirow{2}{*}{ Herederos } & Absolutos & 46 & 24 & 39 & 109 \\
\hline & Relativos & $42.20 \%$ & $22.02 \%$ & $35.78 \%$ & $100 \%$ \\
\hline
\end{tabular}

Fuentes: elaboración propia; CoInPI, 2007.

Cuadro 5. Tipología de representaciones de la profesión de los jóvenes de nuevo ingreso de la carrera de Médico Cirujano, U.V., 2007

\begin{tabular}{|c|c|c|c|c|c|}
\hline $\begin{array}{c}\text { Representaciones } \\
\text { Capital cultural }\end{array}$ & & $\begin{array}{l}\text { 1. Filántropos } \\
\text { privados }\end{array}$ & $\begin{array}{l}\text { 2. Generosos } \\
\text { escépticos }\end{array}$ & $\begin{array}{l}\text { 3. Altruistas } \\
\text { ostensibles }\end{array}$ & Total \\
\hline \multirow{2}{*}{ Héroes } & Absolutos & 5 & 16 & 19 & 40 \\
\hline & Relativos & $12.50 \%$ & $40.00 \%$ & $47.50 \%$ & $100 \%$ \\
\hline \multirow{2}{*}{ Pobres exitosos } & Absolutos & 6 & 14 & 23 & 43 \\
\hline & Relativos & $13.95 \%$ & $32.56 \%$ & $53.49 \%$ & $100 \%$ \\
\hline \multirow{2}{*}{ Herederos } & Absolutos & 12 & 47 & 88 & 147 \\
\hline & Relativos & $8.16 \%$ & $31.97 \%$ & $59.86 \%$ & $100 \%$ \\
\hline
\end{tabular}

Fuente: Fuentes: elaboración propia; CoInPI, 2007. 
Gráfica 1. Clúster de capital cultural. Enfermería y Médico Cirujano, U.V., 2007

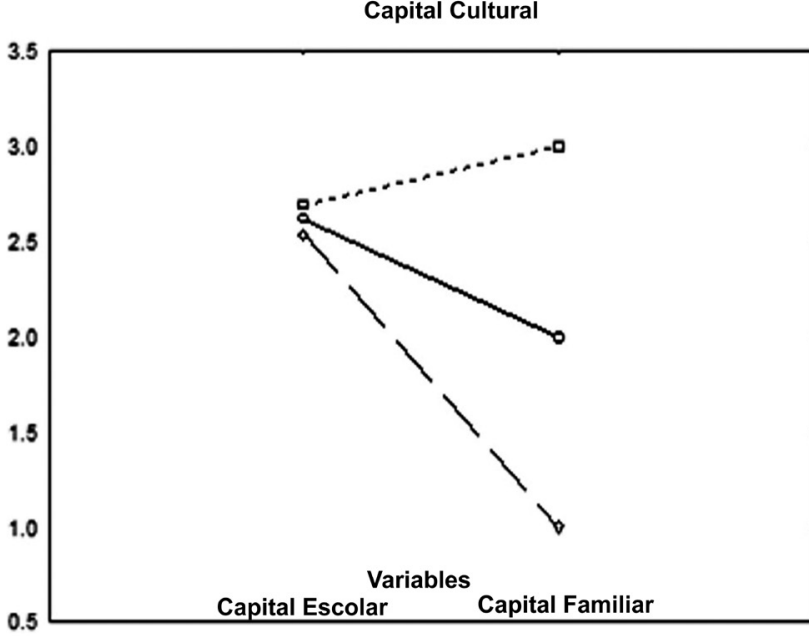

Fuente: elaboración propia con base en COINPI, 2007

Gráfica 1. Clúster de capital cultural. Enfermería y Médico Cirujano, U.V., 2007

Clúster de Representaciones Profesionales Gráfica de medidas para casa grupo

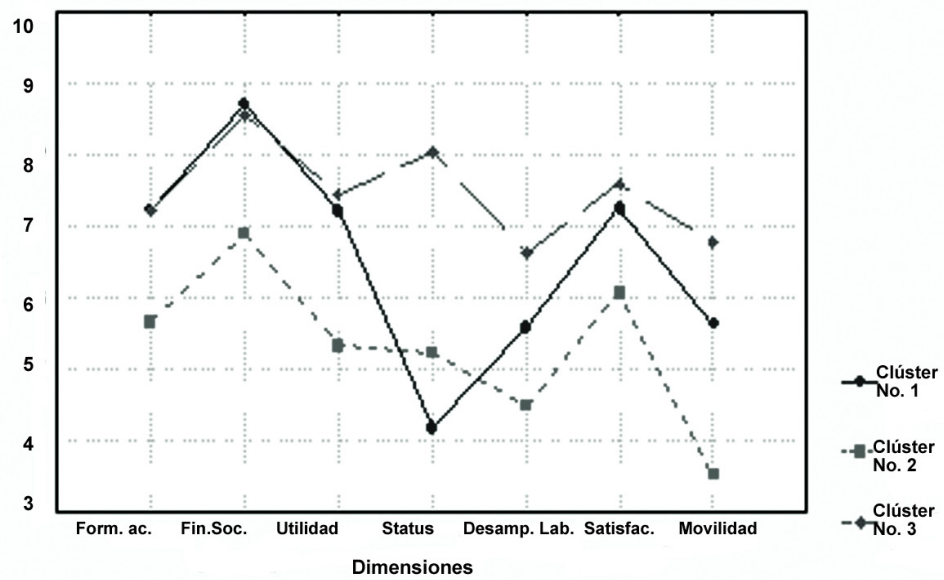

Fuente: elaboración propia con base en COINPI, 2007 